\title{
A Utilização do QSG-12 na População Geral: Estudo de sua Validade de Construto ${ }^{1}$
}

\author{
Valdiney V. Gouveia ${ }^{2}$, Sandra Souza da S. Chaves, Isabel Cristina Possatti de Oliveira ${ }^{3}$, \\ Mardonio Rique Dias, Rildésia S. V. Gouveia e Palloma R.de Andrade \\ Universidade Federal da Paraíba
}

\begin{abstract}
RESUMO - O presente estudo procurou conhecer a validade de construto do QSG-12 na população geral, não clínica. Compuseram a amostra 306 voluntários de diferentes bairros de João Pessoa, com idade média de 34 anos $(D P=13,8$; amplitude de 18 a 84 anos), igualmente distribuídos quanto ao sexo (homens $=152$; mulheres $=154$ ), com escolaridade predominante equivalente ao ensino médio (42,5\%). Estes responderam três medidas: Escala de Satisfação com a Vida, Escala de Afetos Positivos e Negativos e Questionário de Saúde Geral (QSG-12). Os resultados indicaram ser mais adequado considerar esta última medida como bifatorial, representada pelos fatores ansiedade $(\alpha=0,66)$ e depressão $(\alpha=0,81)$. Conforme esperado, tais fatores se correlacionaram com a Escala de Satisfação com a Vida e a Escala de Afetos Positivos e Afetos Negativos, demonstrando a validade convergente do $Q S G$-12. Estes resultados são discutidos à luz dos estudos previamente realizados, sugerindo-se a possibilidade de considerar uma estrutura multidimensional para entender a saúde mental na população geral.
\end{abstract}

Palavras-chave: QSG-12; saúde mental; bem-estar; satisfação com a vida; afetos.

\section{The Use of the GHQ-12 in a General Population: a Study of its Construct Validity}

\begin{abstract}
This study aimed to know the GHQ-12's construct validity in a general, non clinical population. 306 volunteers from different districts in Joao Pessoa, with a mean age of 34 years ( $S D=13.8$; ranging from 18 to 84 ), equally distributed with regards to gender (152 men; 154 woman), predominantly high school students (42.5\%) participated in the study. They answered a questionnaire in which three measures were incorporated: Satisfaction with Life Scale, Positive and Negative Affects Scale, and General Health Questionnaire (GHQ-12). Results indicated that this measure can be considered as bifactorial, with anxiety $(\alpha=.66)$ and depression $(\alpha=.81)$ as the two factors. As expected, both factors correlated with the Satisfaction with Life Scale and the Positive and Negative Affects Scale, indicating the construct validity of the GHQ-12. These results are discussed and compared with results of previous studies suggesting a multidimensional structure for understanding mental health in the general population.
\end{abstract}

Key words: GHQ-12; mental health; well-being; satisfaction with life; affects.

De acordo com Kleinmand e Cohen (1997), compilando dados da Organização Mundial da Saúde, tem se registrado, nos últimos anos, um aumento substancial de pessoas que se queixam de sintomas que poderiam ser caracterizados como típicos de doenças mentais, entre os quais a ansiedade e a depressão. Não obstante, advertem estes mesmos autores, tais sintomas são muitas vezes confundidos com problemas orgânicos, sendo tratados erroneamente. Uma solução prática para resolver estas dificuldades é apresentada pela American Psychiatry Association, centrando-se em esquemas e categorizando as pessoas em função dos sintomas mais proeminentes. Tais esquemas diagnósticos são baseados em descrições constantes no Diagnostic and Statistical Manual of Mental Disorders (DSM-IV, 1995). Em geral, os critérios básicos para o diagnóstico são a presença de sintomas, sua prolongada duração e o prejuízo do funcionamento psíquico.

Apesar do valor heurístico e até mesmo prático de classificações, como as propostas no DSM-IV (1995), a

1 Pesquisa realizada com apoio do CNPq através de bolsa de Produtividade em Pesquisa concedida ao primeiro e quarto autores.

2 Endereço: Universidade Federal da Paraíba, CCHLA, Departamento de Psicologia, 58051-900 João Pessoa, PB, Brasil. E-mail: vvgouveia@uol.com.br ou vgouveia@cchla.ufpb.br

3 In memoriam $(08 / 12 / 2002)$. sintomatologia é identificada a partir de observações do comportamento do paciente e de queixas levadas ao consultório. De modo geral, estas são pouco precisas e difusas, reunindo e misturando tanto fatores físicos como psíquicos da saúde. Percebe-se, portanto, a necessidade de contar com medidas objetivas para avaliar com exclusividade a saúde mental das pessoas, principalmente entre aquelas da população geral (Benjamin, Decalmer \& Haran, 1982; Pasquali, Gouveia, Andriola, Miranda \& Ramos, 1994; Tarnopolsky, Hand, Mclean, Roberts \& Wiggins, 1979). Neste caso, uma qualidade apreciável do instrumento deveria ser sua brevidade. As entrevistas têm seu valor, porém nem sempre são aplicáveis, principalmente quando se trata de efetuar estudos epidemiológicos em comunidades.

Com o propósito de detectar doenças psiquiátricas não severas, isto é, não psicóticas, Goldberg (1972) propôs um instrumento auto-aplicável, tipo lápis e papel, denominado de Questionário de Saúde Geral (QSG). Inicialmente este questionário continha 60 itens, sendo posteriormente apresentado em versões alternativas, mais breves, contendo 30 , 20 e, finalmente, 12 itens, sem comprometimento de sua confiabilidade (Banks \& cols., 1980; Jones \& Murphy, 1979). Esta última versão compreende um dos instrumentos mais utilizados para medir o bem-estar psicológico, principalmente 
em estudos ocupacionais (Borges \& Argolo, 2002; GonzálezRomá \& Espejo, 1993).

No Brasil, o QSG-12 despertou o interesse de alguns pesquisadores (Oliveira \& Dias, 2002; Wagner, Ribeiro, Arteche $\&$ Bornholdt, 1999), existindo ao menos dois estudos que procuram comprovar sua validação de construto (Borges \& Argolo, 2002; Sarriera, Schwarcz \& Câmara, 1996). Ambos, não obstante, centraram-se predominantemente em amostras específicas (bancários, profissionais da saúde, jovens), adotando procedimentos estatísticos estritamente exploratórios para conhecer sua estrutura interna. Além do mais, não avaliaram outros tipos de validade que poderiam atestar a adequação desta medida, como a validade convergente.

Neste contexto, pretende-se aqui contribuir para a validação do QSG-12, procurando tanto comprovar sua dimensionalidade como sua validade convergente com respeito a outros indicadores de saúde mental. Portanto, estes aspectos necessitam ser mais diretamente considerados.

\section{Dimensionalidade do Questionário de Saúde Geral}

Em sua versão com 60 itens, tanto original (Goldberg, 1972) como brasileira (Pasquali \& cols., 1994), o QSG se revela multifatorial. Neste último caso, comprovou-se um fator geral, denominado de severidade de doença mental, reunindo os 60 itens e apresentando índice de consistência interna (Alfa de Cronbach, $\alpha$ ) de 0,95. Cinco fatores específicos puderam ser adequadamente interpretados, com consistência interna e número de itens entre parênteses: estresse psíquico $(\alpha=0,89$; 13 itens), desejo de morte ( $\alpha=0,89 ; 8$ itens), desconfiança no desempenho ( $\alpha=0,89 ; 17$ itens), distúrbios do sono ( $\alpha$ $=0,80 ; 6$ itens) e distúrbios psicossomáticos $(\alpha=0,83 ; 10$ itens). Cabe ressaltar que esta estrutura foi observada com uma amostra homogênea de estudantes, com idades predominantemente inferiores aos 30 anos $(74,8 \%)$. Como os próprios autores desta pesquisa revelam, não se pode descartar uma variabilidade nesta estrutura quando são consideradas pessoas mais maduras, que enfrentam diretamente o drama do desemprego e os desgastes da vida diária.

Quanto ao QSG-12, os resultados de diversas pesquisas não têm sido consistentes em relação a sua estrutura fatorial. A discussão fundamental vem sendo sobre a adequação dos modelos uni e bifatorial desta medida. González-Romá e Espejo (1993), em uma amostra de 112 policiais espanhóis, empregando a análise fatorial confirmatória, observaram que o modelo bifatorial, composto por ansiedade $(\alpha=0,76$; 4 itens) e depressão ( $\alpha=0,83 ; 8$ itens), foi mais adequado do que o unifatorial, denominado de saúde geral $(\alpha=0,87$; 12 itens), $\Delta \chi(1)=6,15, p<0,05$. O modelo bifatorial, não obstante, apresentou índices de ajuste aos dados abaixo do que seria recomendado (Joreskög \& Sörbom, 1989; Rhee, Uleman \& Lee, 1996): $A G F I=0,77$ e $R S M R=0,08$.

Sarriera, Scwarcz e Câmara (1996) avaliaram a estrutura do QSG-12 em uma amostra brasileira. Estes autores consideraram a participação de 563 jovens, com idades entre $16 \mathrm{e}$ 24 anos $(M=19, D P=2,47)$, tendo realizado uma análise de Componentes Principais, com rotação varimax, que revelou a presença de três fatores específicos, cada um com quatro itens, interpretados como segue: auto-estima $(\alpha=0,66)$, depressão $(\alpha=0,68)$ e auto-eficácia percebida $(\alpha=0,54)$. Neste estudo, não se encontra referência às saturações dos itens no fator geral de saúde mental, porém foi calculada a consistência interna para o conjunto total de 12 itens desta medida, tendo sido observado o índice de 0,80 .

Borges e Argolo (2002) também comprovaram a estrutura fatorial do QSG-12. Para tanto, consideraram uma amostra de 158 desempregados e 288 empregados de Natal (RN), sendo que destes 152 eram bancários e 136 profissionais da saúde. Com suas respostas, realizaram uma análise Fatorial dos Eixos Principais, com rotação oblimin, admitindo a correlação entre os prováveis fatores específicos resultantes desta medida. Esta análise foi efetuada tanto para a amostra total $(n=446)$, como para as de desempregados e empregados, tendo revelado consistentemente a presença de dois fatores, interpretados sempre nos mesmos termos. Considerando a amostra geral, a consistência interna destes fatores apresentou os seguintes índices: auto-eficácia $(\alpha=0,85 ; 9$ itens) e depressão e esgotamento emocional ( $\alpha=0,75 ; 3$ itens). Um fator geral, reunindo todos os 12 itens do QSG, foi também computado. Embora não tenham sido apresentados os dados da análise fatorial com critério de extração de um único fator, estes autores indicam que os coeficientes Alfas de Cronbach, para esta estrutura unifatorial, ficaram acima de $0,80 \mathrm{em}$ todas as subamostras consideradas.

Em resumo, concordando com Borges e Argolo (2002),

\begin{abstract}
"nos estudos nacionais e internacionais já realizados sobre o QSG-12, há bastante convergência sobre as avaliações da sua consistência interna e validade enquanto um questionário unifatorial, porém, ainda persistem discussões e dúvidas sobre a adequação de utilizá-lo na mensuração dos subfatores ou fatores primários (dois ou três?)" (p. 19).
\end{abstract}

Além do mais, em geral, tais estudos carecem de comparações entre os diferentes modelos fatoriais; unicamente se limitam a propô-los ou descrevê-los. As pesquisas que efetivamente testaram as estruturas uni e bifatorial, o fizeram com amostras muito específicas e reduzidas, como foi o caso da investigação realizada por González-Romá e Espejo (1993). Porém, existem ainda outros problemas: (a) o uso de análises fatoriais exploratórias, (b) a não clareza sobre o tipo de rotação dos fatores, embora se assuma a presença de um fator geral, e (b) a escassez de dados sobre a validade convergente dos fatores geral e específicos. Este último aspecto é tratado a seguir.

\section{Convergência de Medidas de Saúde Mental}

Não foram encontrados muitos estudos sobre a validade convergente do QSG-12. É possível que o estudo mais extenso já desenvolvido tenha sido realizado por Compton, Smith, Cornish e Qualls (1996), os quais procuraram avaliar a estrutura fatorial de diversas medidas de saúde mental. A propósito, consideraram uma amostra de 338 jovens que responderam 18 instrumentos que avaliavam este construto. Com as respostas destes, efetuaram uma análise de Componentes Principais, tendo encontrado dois fatores de primeira ordem, definidos como bem-estar subjetivo e crescimento pessoal. No primeiro fator, figuraram medidas de felicida- 
de, satisfação com a vida, sentido de coerência, equilíbrio afetivo, qualidade de vida, otimismo, bem-estar psicológico, positividade defensiva e autonomia. Entre as medidas do segundo fator estavam: abertura à experiência, maturidade, auto-realização, vigor e promoção "autoenganosa". O bemestar psicológico, no primeiro fator, foi medido através do QSG-12, sugerindo que este se correlaciona com os afetos e a satisfação com a vida.

Em uma revisão sobre os estudos que atestam a adequação psicométrica da Escala de Satisfação com a Vida, Pavot e Diener (1993) destacaram a correlação da pontuação nesta escala com aquelas obtidas para os fatores ansiedade $(r=$ $-0,54, p<0,01)$ e depressão $(r=-0,55, p<0,01)$, avaliados através do Symptom Checklist-90. Este instrumento, como o QSG-12, avalia a saúde mental, sugerindo ser este um dos componentes centrais do bem-estar subjetivo experimentado pelas pessoas.

Em resumo, os estudos realizados com o QSG - 12 ainda são incipientes no que diz respeito a sua estrutura fatorial; parece haver mais consenso sobre sua natureza unifatorial, embora sejam também sugeridos modelos bi e, inclusive, multifatorial. Portanto, este estudo tem como objetivo principal contribuir para dirimir esta dúvida, além de permitir avaliar em que medida este questionário é adequado enquanto um indicador de bem-estar subjetivo. Para atender a estes propósitos, procurou-se contar com uma amostra da população geral da cidade de João Pessoa, evitando tratar com populações específicas, como as de estudantes e trabalhadores.

\section{Método}

\section{Amostra}

Participaram desta pesquisa 306 pessoas da população geral, provenientes de diferentes bairros de João Pessoa. De acordo com a classificação sócio-econômica destes bairros, realizada por Lopes de Andrade (2001), observou-se a seguinte distribuição: classe baixa $(37,9 \%)$, classe média $(35,9 \%)$ e classe alta $(26,1 \%)$. Estes participantes foram igualmente distribuídos em relação ao gênero, com idades variando de 18 a 84 anos $(M=34,2 ; D P=13,8)$. A maioria indicou ser casada ou com um relacionamento prévio duradouro $(55,9 \%)$, com escolaridade equivalente ao ensino médio $(42,5 \%)$ e religião católica $(64,1 \%)$, afirmando ser bastante ou muito religiosa $(59,8)$.

\section{Instrumentos}

Os participantes foram solicitados a responder a um conjunto de cinco medidas, três das quais são descritas aqui por sua relevância para contemplar os objetivos deste estudo:

Questionário de Saúde Geral (QSG-12). Este instrumento corresponde a uma versão abreviada do Questionário de Saúde Geral de Goldberg (1972), adaptado para o Brasil por Pasquali e cols. (1994). Como sua abreviação indica, a versão que é utilizada neste estudo se compõe de 12 itens (por exemplo: Você tem se sentido pouco feliz e deprimido?; Você tem perdido a confiança em si mesmo?). Cada item é respondido em termos do quanto a pessoa tem experimentado os sintomas descritos, sendo suas respostas dadas em uma escala de quatro pontos. No caso dos itens que negam a saúde mental (por exemplo: Suas preocupações lhe têm feito perder muito sono?; Tem se sentido pouco feliz e deprimido?), as alternativas de resposta variam de 1 (Absolutamente, não) a 4 (Muito mais que de costume); no caso dos itens afirmativos (por exemplo: Tem se sentido capaz de tomar decisões?; Tem podido concentrar-se bem no que faz?), as respostas foram de 1 (Mais que de costume) a 4 (Muito menos que de costume). Os itens negativos foram invertidos, de modo que a maior pontuação total nesta medida indica melhor nível de saúde mental. Como antes indicado, os estudos realizados no Brasil, inclusive sem definir uma estrutura fatorial única, concordam sobre a existência de um fator geral, com Alfa de Cronbach variando de 0,80 (Sarriera, Schwarcz \& Câmara, 1996) a 0,88 (Borges \& Argolo, 2002).

Escala de Afetos Positivos e Negativos. Esta medida foi originalmente proposta por Diener e Emmons (1984, conforme citado por Reis, Gable, Roscoe \& Ryan, 2000) para avaliar a valência dos afetos, tendo sido realizados estudos recentes que comprovam a adequação dos seus parâmetros psicométricos (Omodei \& Wearing, 1990; Sheldon \& Kasser, 1995). Esta escala se compõe de nove adjetivos, sendo quatro positivos e cinco negativos. Os afetos positivos são: feliz, alegre, satisfeito e divertido; e os negativos são: deprimido, preocupado, frustrado, raivoso e infeliz. Procurando equilibrar o número de adjetivos para ambos tipos de afetos, foi acrescentado nesta pesquisa um adjetivo para o afeto positivo: otimista. Este instrumento avalia o quanto o participante tem experimentado cada uma destas emoções nos últimos dias. Os itens são respondidos em uma escala de sete pontos, com os extremos 1 (Nada) e 7 (Extremamente).

Escala de Satisfação com a Vida. Esta medida foi proposta originalmente por Ed Diener, tendo sido realizados estudos recentes que comprovam a adequação dos seus parâmetros psicométricos. Por exemplo, Pavot e Diener (1993) descrevem uma consistência interna, Alfa de Cronbach, de 0,87; sua estabilidade temporal, considerando um período de dois meses, foi de 0,82 . Estes autores realizaram também uma análise fatorial com os dados obtidos, tendo observado um único fator que explicou $66 \%$ da variância total. Compõe-se de cinco itens, como os que seguem: estou satisfeito com minha vida e as condições da minha vida são excelentes, os quais são respondidos em uma escala tipo Likert, variando de 1 (Discordo Totalmente) a 7 (Concordo Totalmente).

Além destas medidas, um conjunto de 12 perguntas de natureza demográfica foi inserido com o fim de caracterizar a amostra (por exemplo, idade, sexo, classe social, escolaridade, religião, dentre outras).

\section{Procedimento}

Dois colaboradores foram treinados em relação ao procedimento necessário para aplicar os instrumentos. Estes, juntamente com o pesquisador principal, visitaram diferentes bairros de João Pessoa, selecionando as residências e locais públicos (por exemplo, praças e mercados) para a coleta dos dados. As pessoas foram solicitadas a responder voluntariamente a um questionário, identificado como uma medida de atitudes e pensamentos em relação à vida. Estas foram informadas que o objetivo da pesquisa era de nature- 
za científica, evitando que fosse confundida com propósitos políticos ou econômicos. O tempo médio para responder aos questionários foi de 25 minutos. Procurou-se entrevistar uma pessoa com mais de 18 anos, cujo aniversário fosse o mais recente entre aqueles que estavam presentes na casa. Quando não se obtinha a colaboração das pessoas de uma residência, passava-se à vizinha. Como se tratava de uma pesquisa cuja variável de interesse pode sofrer influências externas, como festas comemorativas de fim de ano, carnaval etc., procurouse coletar os dados em períodos que não correspondessem a estas datas. Neste sentido, a pesquisa foi realizada nos primeiros vinte dias do mês de Novembro de 2001.

\section{Análise de dados}

A análise fatorial confirmatória foi realizada com o programa LISREL 8. Embora existem múltiplos indicadores estatísticos para decidir sobre a adequação dos modelos teóricos, os quatro seguintes são provavelmente os mais conhecidos (Joreskög \& Sörbom, 1989):

$\chi$ (Qui-quadrado) - comprova a probabilidade de um modelo se ajustar aos dados. Sua significância sugere discrepância do modelo em relação aos dados.

"Bondade de Ajuste Subjetiva" - compreende uma razão entre o

$\chi$ e os graus de liberdade (g.l.). Assume-se que seu valor deva ser igual ou inferior a 5,00 para que o modelo seja considerado adequado (Byrne, 1989).

GFI - índice de bondade de ajuste, expressando uma medida de variabilidade explicada pelo modelo. Um valor de 0,80 ou mais indica que o modelo se ajusta aos dados (Rhee, Uleman \& Lee, 1996).

RMSR - corresponde à raiz quadrada média residual. Um valor próximo de zero indica que o modelo teórico se ajusta aos dados, uma vez que seus residuais (diferença entre o modelo e os dados) se aproximam de zero (Joreskög \& Sörbom, 1989).

\section{Resultados}

\section{As Medidas de Afetos e Satisfação com a Vida}

Embora não seja o propósito principal do presente estudo, com o fim de avaliar a validade convergente do QSG-12 com as medidas de bem-estar subjetivo, é necessário demonstrar previamente a adequação destas. Neste sentido, a seguir serão apresentados os resultados correspondentes a sua validade de construto.

\section{Escala de Afetos Positivos e Negativos}

Comprovou-se inicialmente a adequação de se realizar uma análise fatorial, tendo sido observados os seguintes indicadores que a atestam: $K M O=0,84$; e Teste de Esfericidade de Bartlett,

$\chi^{2}(45)=1131,87, p<0,001$. Neste sentido, realizou-se uma análise PC (Principal Components), com rotação oblimin, tendo os resultados indicado a existência de dois fatores com eigenvalues superiores a 1, explicando conjuntamente
Tabela 1. Estrutura Fatorial da Escala de Afetos Positivos e Negativos

\begin{tabular}{llcc}
\hline \multirow{2}{*}{ Itens } & \multirow{2}{*}{ Conteúdo } & \multicolumn{2}{c}{ Fatores } \\
\cline { 3 - 4 } & & I & II \\
\hline 10 & Alegre & $0,91^{*}$ & 0,02 \\
06 & Divertido & $0,87^{*}$ & 0,16 \\
01 & Feliz & $0,71^{*}$ & $-0,20$ \\
03 & Satisfeito & $0,68^{*}$ & $-0,20$ \\
08 & Otimista & $0,44^{*}$ & $-0,11$ \\
05 & Raivoso & 0,22 & $0,79^{*}$ \\
04 & Frustrado & $-0,02$ & $0,76^{*}$ \\
02 & Deprimido & $-0,17$ & $0,67^{*}$ \\
09 & Infeliz & $-0,25$ & $0,63^{*}$ \\
07 & Preocupado & $-0,18$ & $0,57^{*}$ \\
\hline Eigenvalue & 4,28 & 1,43 \\
\hline$\%$ Variância Explicada & 42,8 & 14,3 \\
\hline \multicolumn{2}{l}{ Alfa de Cronbach $(\alpha)$} & 0,81 & 0,78 \\
\hline
\end{tabular}

* |0,30| (carga fatorial mínima considerada para interpretação dos fatores). Identificação dos Fatores: $\mathrm{I}=$ Afetos Positivos; e II = Afetos Negativos.

$57,1 \%$ da variância total. Os principais resultados desta análise podem ser vistos na Tabela 1.

Considerando um valor absoluto de 0,30 da carga fatorial para interpretar o fator, é possível denominá-los como segue:

Fator I. É composto por cinco itens, com saturações variando entre 0,44 (otimista) e 0,91 (alegre). Apresentou eigenvalue de 4,28, explicando $42,8 \%$ da variância total, com índice de consistência interna (Alfa de Cronbach, $\alpha$ ) de 0,81. Claramente, é possível defini-lo como afeto positivo.

Fator II. Cinco itens formam este fator, com saturações variando de 0,57 (preocupado) a 0,79 (raivoso). Seu eigenvalue foi de 1,43, explicando $14,3 \%$ da variância total, com índice de consistência interna (a) de 0,78. É inequívoca sua denominação de afeto negativo.

Uma análise fatorial confirmatória permitiu observar a adequação desta estrutura bifatorial:

$\chi(34)=189,84, p<0,01$;

$\chi /$ g.l. $=5,59 ;$ RMRS $=0,06 ; G F I=0,89 ; A G F I=0,81$. Todas as cargas fatoriais $(\lambda \lambda$, Lambdas) foram estatisticamente diferentes de zero $(0 ; t>1,96, p<0,05)$.

\section{Escala de Satisfação com a Vida}

A amostra de itens desta medida foi considerada adequada para obter uma solução fatorial: $K M O=0,82$ e Teste de Esfericidade de Bartlett,

$\chi^{2}(10)=375,43, p<0,001$. A propósito, realizou-se uma análise PC, sem fixar o número de fatores a serem extraídos. Esta análise permitiu identificar um único fator com eigenvalue de 2,67, explicando 53,2\% da variância total, correspondendo à dimensão de Satisfação com a Vida. Suas saturações variaram de 0,61 (Se pudesse viver uma segunda vez, não mudaria quase nada na minha vida) a 0,79 (As condições da minha vida são excelentes), com consistência interna $(\alpha)$ de 0,72. Estes resultados são sumarizados na Tabela 2.

Uma análise fatorial confirmatória permitiu comprovar a adequação desta estrutura fatorial:

$\chi(5)=5,02, p>0,05$ 
Tabela 2. Estrutura Fatorial da Escala de Satisfação com a Vida

\begin{tabular}{|c|c|c|}
\hline Itens & Conteúdo & Fator \\
\hline 2. & As condições da minha vida são excelentes & $0,79 *$ \\
\hline 3. & Estou satisfeito com minha vida & $0,78^{*}$ \\
\hline 1. & $\begin{array}{l}\text { Na maioria dos aspectos minha vida é próxima ao } \\
\text { meu ideal }\end{array}$ & $0,75^{*}$ \\
\hline 4. & $\begin{array}{l}\text { Dentro do possível, tenho conseguido as coisas } \\
\text { importantes que quero na vida }\end{array}$ & $0,70^{*}$ \\
\hline 5. & $\begin{array}{l}\text { Se pudesse viver uma segunda vez, não mudaria } \\
\text { quase nada na minha vida }\end{array}$ & $0,61^{*}$ \\
\hline \multicolumn{2}{|c|}{ Eigenvalue } & 2,67 \\
\hline \multicolumn{2}{|c|}{ \% Variância Explicada } & 53,2 \\
\hline \multicolumn{2}{|c|}{ Alfa de Cronbach } & 0,72 \\
\hline
\end{tabular}

$\chi /$ g.l. $=1,00 ; R M R S=0,02 ; G F I=0,99 ; A G F I=0,98$. Como esperado, todas as cargas fatoriais $(\lambda \lambda)$ foram estatisticamente diferentes de zero $(0 ; t>1,96, p<0,05)$.

De acordo com o antes exposto, comprova-se a adequação dos parâmetros psicométricos das medidas de afetos e satisfação com a vida, o que justificará mais adiante considerálas como referentes para avaliar a validade convergente do QSG-12. Previamente, não obstante, é preciso igualmente conhecer a validade de construto desta medida, o que se procura fazer a seguir.

\section{Validade de Construto do QSG-12}

Como ficou evidenciado na introdução, a solução unifatorial, ao menos no Brasil, parece mais evidente do que a bifatorial. Não obstante, quando são usados procedimentos de análise fatorial exploratória, diferentes soluções têm sido observadas. Neste sentido, antes mesmo de efetuar uma análise fatorial confirmatória, apresentam-se os resultados derivados da exploração da estrutura fatorial dos dados aqui obtidos.

\section{Análise Fatorial Exploratória}

Considerou-se inicialmente a adequação de se efetuar uma análise fatorial com o conjunto de itens desta medida, o que foi plenamente corroborado: $K M O=0,85$ e Teste de Esfericidade de Bartlett,

$\chi^{2}(66)=1066,90, p<0,001$. Empregando a análise PAF (Principal Axis Factoring), com rotação oblíqua (Direct Oblimin), procedimentos utilizados por Borges e Argolo (2002), encontraram-se três fatores com eigenvalues superiores a 1 . Estes explicaram conjuntamente $44,8 \%$ da variância total. Os principais resultados desta análise são observados na Tabela 3.

Tomando como referência a carga fatorial mínima de 0,30 para interpretar cada fator, as seguintes etiquetas pareceram mais adequadas:

Fator I. Reuniu cinco itens, com saturações de 0,46 (Tem podido concentrar-se bem no que faz?) a 0,67 (Sente-se razoavelmente feliz considerando todas as circunstâncias?). Seu eigenvalue foi de 4,49, explicando $33 \%$ da variância total, com índice de consistência interna $(\alpha)$ de 0,72 . Esta estrutura sugere denominar este fator de depressão.

Fator II. Três itens representaram este fator, com saturações entre -0,57 (Suas preocupações lhe têm feito perder o sono?) e -0,80 (Tem notado que está constantemente agoniado e tenso?). Este apresentou eigenvalue de 1,33, explicando 6,9\% da variância total, com índice de consistência interna (a) de 0,76. Parece inequívoca sua denominação de ansiedade.

Fator III. Este fator concentrou cinco itens, com saturações variando de $-0,30$ (Tem se sentido capaz de tomar decisões?) a -0,72 (Tem pensado que você é uma pessoa que não serve para nada?), com eigenvalue de 1,15 , correspondendo à explicação de 4,9\% da variância total. Seu índice de consistência interna (a) foi de 0,76 . Decidiu-se denominá-lo de auto-eficácia.

A matriz de correlações destes fatores revelou que todos estão correlacionados entre si, com $r$ variando de 0,40 (Fator

Tabela 3. Estrutura Fatorial do Questionário de Saúde Geral (QSG-12)

\begin{tabular}{|c|c|c|c|c|}
\hline \multirow{2}{*}{ Itens } & \multirow{2}{*}{ Conteúdo Abreviado } & \multicolumn{3}{|c|}{ Fatores } \\
\hline & & $\mathbf{I}$ & II & III \\
\hline 12 & Sente-se razoavelmente feliz & $0,67^{*}$ & $-0,00$ & 0,10 \\
\hline 08 & Tem sido capaz de enfrentar problemas & $0,55^{*}$ & 0,11 & $-0,22$ \\
\hline 04 & Tem sido capaz de tomar decisões & $0,50^{*}$ & 0,11 & $-0,30 *$ \\
\hline 07 & Tem sido capaz de desfrutar de atividades & $0,48^{*}$ & $-0,20$ & 0,00 \\
\hline 01 & Tem podido concentrar-se no que faz & $0,46^{*}$ & $-0,19$ & 0,00 \\
\hline 05 & Tem notado que está agoniado & 0,00 & $-0,80 *$ & 0,00 \\
\hline 09 & Tem se sentido pouco feliz e deprimido & 0,00 & $-0,64 *$ & $-0,16$ \\
\hline 02 & Suas preocupações o fazem perder sono & $-0,01$ & $-0,57 *$ & $-0,01$ \\
\hline 11 & Tem pensado que não serve para nada & $-0,12$ & $-0,01$ & $-0,72 *$ \\
\hline 10 & Tem perdido confiança em si mesmo & 0,01 & $-0,15$ & $-0,62 *$ \\
\hline 03 & Tem sentido que tem papel útil na vida & 0,21 & 0,00 & $-0,55^{*}$ \\
\hline 06 & Tem sensação de não superar dificuldades & 0,14 & $-0,22$ & $-0,35^{*}$ \\
\hline \multicolumn{2}{|c|}{ Eigenvalue } & 4,49 & 1,33 & 1,45 \\
\hline \multicolumn{2}{|c|}{ \% Variância Explicada } & 33,0 & 6,9 & 4,9 \\
\hline \multicolumn{2}{|c|}{ Alfa de Cronbach $(\alpha)$} & 0,72 & 0,76 & 0,76 \\
\hline
\end{tabular}


III versus Fator II) a -0,51 (Fator I versus Fator III), $p<0,001$. Deste modo, optou-se por extrair um único fator para o conjunto de itens que compõem o QSG-12, tendo sido observadas saturações no intervalo de 0,45 (Suas preocupações lhe têm feito perder muito sono?) a 0,67 (Tem perdido a confiança em si mesmo?), com consistência interna (a) de 0,84. Este fator geral, que pode ser denominado de saúde mental ou bem-estar, apresentou eigenvalue de 3,83, explicando $31,9 \%$ da variabilidade de respostas dos participantes.

Tendo sido explorada a estrutura fatorial do QSG-12, é necessário, então, comprovar a adequação dos dois principais modelos teóricos que têm sido referidos na literatura, correspondendo às estruturas unifatorial versus bifatorial.

\section{Comprovação dos Diferentes Modelos}

Para efetuar a análise fatorial confirmatória, considerouse a matriz de correlações (r de Pearson) como entrada, tendo sido adotado o estimador GL (Generalized Least Squares). Fixando os parâmetros a serem estimados de acordo com os modelos teóricos aqui testados, comprovaram-se as saturações apresentadas na Tabela 4.

Como é possível observar, todas as saturações são estatisticamente diferentes de zero $(t>1,96, p<0,05)$, indicando sua adequação no fator correspondente. O leitor interessado poderá ter acesso aos índices de modificação para redefinição dos itens em cada fator. Em termos da proposta do presente estudo, cabe averiguar o melhor modelo entre aqueles que foram teorizados. $\mathrm{O}$ modelo bifatorial apresentou $\chi(53)=$ 351,21 , com índice subjetivo de ajuste $(\chi / \mathrm{g} .1)$ de 6,62 ; seu RMSR foi de 0,15 e GFI de 0,81 . No caso do modelo unifatorial, os seguintes valores foram observados: $\chi(54)=359,42$, índice subjetivo de ajuste $(\chi / g .1)=6,66, R M S R=0,15$ e $G F I$ $=0,80$. Neste sentido, o primeiro modelo parece ligeiramente mais adequado do que este último. Considerando a diferença entre os qui-quadrados e os respectivos graus de liberdade destes modelos, confirma-se que esta superioridade é estatisticamente significativa: $\Delta \chi(1)=8,21, p<0,01$.

\section{Validade Convergente do QSG-12 com os Indicadores de Bem-estar Subjetivo}

Considerando os resultados previamente descritos para os modelos teóricos do QSG-12, bem como os índices de consistência interna observados, para os dois fatores específicos e o geral, justifica-se aqui comprovar a validade convergente de tais dimensões com respeito aos indicadores de bem-estar subjetivo empregados neste estudo. Os resultados são apresentados na Tabela 5 .

De acordo com esta tabela, o fator geral (unidimensional) de saúde mental se correlaciona significativamente ( $p$ $<0,001)$ com todos os indicadores de bem-estar subjetivo, a saber: afetos negativos $(r=-0,54)$, afetos positivos $(r=$ $0,54)$ e satisfação com a vida $(r=0,51)$. O mesmo ocor-

Tabela 4. Análise Fatorial Confirmatória do Questionário de Saúde Geral (QSG-12)

\begin{tabular}{|c|c|c|c|c|}
\hline \multirow{3}{*}{ Itens } & \multirow{3}{*}{ Conteúdo Abreviado } & \multicolumn{3}{|c|}{ Dimensão dos Modelos } \\
\hline & & \multicolumn{2}{|c|}{ Bifatorial } & \multirow{2}{*}{$\begin{array}{c}\text { Unifatorial } \\
\text { I }\end{array}$} \\
\hline & & I & II & \\
\hline 11 & Tem pensado que não serve para nada & $0,80^{*}$ & & $0,80^{*}$ \\
\hline 10 & Tem perdido confiança em si mesmo & $0,82 *$ & & $0,83^{*}$ \\
\hline 03 & Tem sentido que tem papel útil na vida & $0,69^{*}$ & & $0,63^{*}$ \\
\hline 09 & Tem se sentido pouco feliz e deprimido & $0,66^{*}$ & & $0,73^{*}$ \\
\hline 04 & Tem sido capaz de tomar decisões & $0,66^{*}$ & & $0,59 *$ \\
\hline 06 & Tem sensação de não superar dificuldades & $0,62 *$ & & $0,63^{*}$ \\
\hline 08 & Tem sido capaz de enfrentar problemas & $0,54 *$ & & $0,49 *$ \\
\hline 12 & Sente-se razoavelmente feliz & $0,46^{*}$ & & $0,45^{*}$ \\
\hline 05 & Tem notado que está agoniado & & $0,62 *$ & $0,68^{*}$ \\
\hline 01 & Tem podido concentrar-se no que faz & & $0,59 *$ & $0,52 *$ \\
\hline 07 & Tem sido capaz de desfrutar de atividades & & $0,58^{*}$ & $0,55^{*}$ \\
\hline 02 & Suas preocupações o fazem perder sono & & $0,45^{*}$ & $0,51 *$ \\
\hline \multicolumn{2}{|c|}{ Alfa de Cronbach } & 0,81 & 0,66 & 0,84 \\
\hline
\end{tabular}

Tabela 5. Correlação dos Fatores do QSG-12 com Indicadores de Bem-Estar Subjetivo.

\begin{tabular}{|c|c|c|c|c|c|c|c|}
\hline & $\mathbf{M}$ & DP & 1 & 2 & 3 & 4 & 5 \\
\hline 1. Saúde Mental & 3,1 & 0,54 & & & & & \\
\hline 2. Depressão & 3,2 & 0,57 & $0,96^{*}$ & & & & \\
\hline 3. Ansiedade & 2,7 & 0,67 & $0,81^{*}$ & $0,62 *$ & & & \\
\hline 4. Satisfação com a Vida & 4,8 & 1,22 & $0,51^{*}$ & $0,47^{*}$ & $0,43^{*}$ & & \\
\hline 5. Afetos Positivos & 4,8 & 1,13 & $0,54 *$ & $0,49 *$ & $0,49 *$ & $0,62^{*}$ & \\
\hline 6. Afetos Negativos & 2,7 & 1,27 & $-0,55^{*}$ & $-0,48 *$ & $-0,55^{*}$ & $-0,51 *$ & $-0,50 *$ \\
\hline
\end{tabular}

Notas: $* p<0,001$ (teste unicaudal). A maior pontuação no QSG-12 e nos seus dois fatores (ansiedade e depressão) indicam um nível mais elevado de saúde mental. 
reu para seus fatores de ansiedade $(\mathrm{r}=-0,55,0,49$ e 0,43 , respectivamente) e depressão ( $r=-0,48,0,49$ e 0,47 , respectivamente). Estes dois fatores de saúde mental também se correlacionaram direta e significativamente entre $\mathrm{si}(\mathrm{r}=$ $0,62, p<0,001)$.

\section{Discussão}

O presente estudo teve como objetivo geral contribuir para o conhecimento dos parâmetros psicométricos do QSG-12 na população brasileira. Espera-se que este tenha sido alcançado; as análises estatísticas efetuadas permitiram comprovar sua validade de construto, apesar dos índices de bondade de ajuste da análise fatorial confirmatória não terem sido tão satisfatórios. Não obstante, não é possível deixar de reconhecer uma de suas prováveis limitações: a natureza da amostra considerada. Embora esta tenha o mérito de não se restringir à população estudantil universitária, o fato de considerar pessoas da população geral que se encontravam em suas casas no momento da realização da pesquisa pode introduzir um viés. Especificamente, é possível que isso indique sua condição de desempregado, principalmente no caso dos homens; este dado deveria ter sido levado em consideração, uma vez que a condição laboral das pessoas tem um papel importante no seu nível de saúde mental (Banks \& cols., 1980; Borges \& Argolo, 2002).

Quanto aos parâmetros psicométricos desta medida, os resultados foram bastante consistentes com aqueles descritos na literatura. Os dados de González-Romá e Espejo (1993) de que o QSG-12 pode ser melhor representado por uma estrutura bifatorial, quando comparada com uma unifatorial, foram plenamente corroborados. Em sua análise fatorial exploratória, Borges e Argolo (2002) puderam igualmente identificar com clareza uma estrutura bifatorial nas respostas de pessoas empregadas e desempregadas, porém apenas um dos seus fatores concordou com aqueles descritos pelos autores antes citados: depressão. $\mathrm{O}$ seu segundo fator foi denominado de auto-eficácia, não correspondendo ao fator ansiedade, objeto de comprovação no estudo de GonzálezRomá e Espejo (1993) e na presente pesquisa.

O fator auto-eficácia surgiu também com bastante nitidez no estudo de Sarriera, Schwarcz e Câmara (1996), tendo sido corroborado na análise fatorial exploratória aqui realizada. A propósito, é necessário destacar que esta análise revelou mais outros dois fatores: ansiedade e depressão. Não fosse pelo primeiro fator dos autores previamente citados, denominado por eles de auto-estima, comprovar-se-ia uma estrutura fatorial comum nestes dois estudos. Não obstante, apesar da divergência observada na denominação dos seus fatores, tais estudos contribuem a que se pense a saúde mental, conforme medida pelo QSG-12, como um construto multidimensional.

A despeito do consenso sobre o questionário analisado ser uma medida unifatorial da saúde mental (Borges \& Argolo, 2002), os resultados aqui descritos impõem alguma restrição. De acordo com as saturações observadas para os seus 12 itens no modelo unifatorial (Tabela 4), uma solução deste tipo é admissível; sem dúvida permite maior parcimônia na descrição do estado de saúde experimentado pelas pessoas, inclusive apresentando consistência interna dentro do que se- ria esperado (Banks \& cols., 1980; González-Romá \& Espejo, 1993). Porém, não retrata os resultados obtidos tanto nesta pesquisa, como nas anteriormente realizadas com amostras da população brasileira, quando não se fixou o número de fatores a serem extraídos nas análises fatoriais (Borges \& Argolo, 2002; Sarriera, Schwarcz \& Câmara, 1996). Portanto, pode-se empregar o QSG-12 como um índice geral de saúde mental, mas se recomenda ter em conta sua natureza multi ou ao menos bifatorial.

Quanto a considerar esta medida como uma indicação válida de bem-estar, os resultados observados aqui são satisfatórios, corroborando os dados de Compton, Smith, Cornish \& Qualls (1996). Tratando com três indicadores de bem-estar (satisfação com a vida, afetos negativos e afetos positivos), comprovou-se a validação convergente do QSG-12. Neste sentido, pode-se assumir que tanto o modelo uni, como o bifatorial, permitem adequadamente expressar o estado de ânimo das pessoas ou propriamente seu nível de esgotamento psicológico (Oliveira \& Dias, 2002). Cabe aqui dar ênfase também a adequação de se utilizar em pesquisas futuras as versões brasileiras da Escala de Satisfação com a Vida (Pavot \& Diener, 1993) e a Escala de Afetos Positivos e Negativos (Diener \& Emmons, 1984, conforme citado por Reis \& cols., 2000), as quais apresentaram parâmetros psicométricos bastantes promissores (Pasquali, 1999).

Em termos do que fazer no futuro, parece relevante replicar a presente pesquisa, considerando pessoas da população geral, mas tendo a informação sobre sua situação laboral. Isso permitirá descartar o provável viés indicado ao começo desta discussão. Em uma eventual replicação, seria recomendável também contar com medidas mais diretas de saúde mental, que enfocassem os fatores de ansiedade e depressão que compõem este questionário. Le Fevre, Devereux, Smith, Lawrie e Cornbleet (1999), por exemplo, consideraram uma medida destes construtos, procurando contrastar sua eficácia com o QSG-12 para o diagnóstico de distúrbios psiquiátricos em um contexto hospitalar. Porém, estes autores não correlacionaram diretamente tais medidas, dificultando avaliar a extensão da sua convergência.

Um fator de auto-eficácia aparece nas análises fatoriais dos dois estudos prévios de adaptação do QSG-12 à realidade brasileira (Borges \& Argolo, 2002; Sarriera, Schwarcz \& Câmara, 1996). No presente foi igualmente identificado este fator, definido por itens, como: Tem pensado que não serve para nada? e Tem perdido confiança em si mesmo?, tendo apresentado consistência interna aceitável $(\alpha=0,76)$. Este aspecto sugere que, ademais da estrutura identificada por González-Romá e Espejo (1993), as respostas dos participantes do Brasil permitem propor um terceiro fator para esta medida. Esperar-se-ia que pesquisas futuras pusessem à prova esta estrutura com três fatores, utilizando, por exemplo, modelos de equações estruturais (Byrne, 1989; Joreskög \& Sörbom, 1989) que permitissem contrastá-los com as soluções uni e bifatorial.

Finalmente, além de ter utilidade em pesquisas científicas, esta medida pode ser útil em alguns contextos práticos. Por exemplo, Borges e Argolo (2002) comentam que "o referido instrumento de medida poderá ser utilizado também como instrumental na atuação profissional na área de administração de pessoas, para desenvolver diagnósticos organizacionais 
e/ou de saúde coletiva numa perspectiva epidemiológica aplicada ao trabalho" (p. 19). Neste contexto, é preciso ressaltar que, dado sua brevidade, poderia ser utilizado também para conhecer o nível de saúde mental das pessoas de determinada comunidade ou populações em risco, assim como servir de instrumento de triagem em contextos ambulatoriais, evitando-se gastos desnecessários em tratamento com problemas erroneamente identificados como de natureza orgânica.

\section{Referências}

Banks, M.H., Clegg, C.W., Jackson, P.R., Kemp, N.J., Stafford, E.M. \& Wall, T.D. (1980). The use of the General Health Questionnaire as an indicator of mental health in occupational studies. Journal of Occupational Psychology, 53, 187-194.

Benjamin, S., Decalmer, P. \& Haran, D. (1982). Community screening for mental illness: a validity study of the General Health Questionnaire. British Journal of Psychiatry, 140, 174-180.

Borges, L. de O. \& Argolo, J.C.T. (2002). Adaptação e validação de uma escala de bem-estar psicológico para uso em estudos ocupacionais. Avaliação Psicológica, 1, 17-27.

Byrne, B.M. (1989). A primer of LISREL: basic applications and programming for confirmatory factor analytic models. New York: Springer-Verlag.

Compton, W.C., Smith, M.L., Cornish, K.A. \& Qualls, D.L. (1996). Factor structure of mental health measures. Journal of Personality and Social Psychology, 71, 406-413.

DSM-IV. (1995). Casos clínicos: Complemento didático para o manual diagnóstico e estatístico de transtornos mentais $\left(4^{\mathrm{a}}\right.$ edição). Porto Alegre: Artes Médicas.

Goldberg, D. (1972). The detection of psychiatric illness by questionnaire. London: Oxford University Press.

González-Romá, V., Lloret, S. \& Espejo, B. (1993). Comparación de los modelos de medida del Cuestionario de Salud General (GHQ-12). Psicológica, 14, 259-268.

Jones, R.A. \& Murphy, E. (1979). Severity of psychiatric disorder and the 30-item General Health Questionnaire. British Journal of Psychiatry, 134, 609-616.

Joreskög, K.G. \& Sörbom, D. (1989). LISREL 7 user's reference guide. Mooresville: Scientific Software.

Kleinman, A. \& Cohen, A. (1997). Psychiatry's global challenge. Scientific American, 276, 86-89.

Le Fevre, P., Devereux, J., Smith, S., Lawrie, S.M. \& Cornbleet, M. (1999). Screening for psychiatric illness in the palliative care inpatient setting: a comparison between the Hospital Anxiety and Depression Scale and the General Health Questionnaire-12. Palliative Medicine, 13, 399-407.

Lopes de Andrade, M.W.C. (2001). A dimensão valorativa do sentido da vida. Dissertação de Mestrado, Universidade Federal da Paraíba, João Pessoa.

Oliveira, I.C.P \& Dias, M.R. (2002). Multiplicidade de papéis da mulher e seus efeitos para o bem-estar psicológico. Psicologia: Reflexão e Crítica, 15, 293-301.

Omodei, M.M. \& Wearing, A.J. (1990). Need satisfaction and involvement in personal projects: toward an integrative model of subjective well-being. Journal of Personality and Social Psychology, 59, 762-769.

Pasquali, L. (1999). Instrumentos psicológicos: manual prático de elaboração. Brasília: LabPAM / IBAPP.

Pasquali, L., Gouveia, V.V., Andriola, W.B., Miranda, F.J. \& Ramos, A.L.M. (1994). Questionário de Saúde Geral de Goldberg (QSG): adaptação brasileira. Psicologia: Teoria e Pesquisa, 10, 421-437.

Pavot, W. \& Diener, E. (1993). Review of the satisfaction with life scale. Psychological Assessment, 5, 164-172.

Reis, H.T., Sheldon, K.M., Gable, S.L., Roscoe, J. \& Ryan, R.M. (2000). Daily well-being: the role of autonomy, competence, and relatedness. Personality and Social Psychology Bulletin, 26, 419-435.

Rhee, E., Uleman, J.S. \& Lee, H.K. (1996). Variations in collectivism and individualism by ingroup and culture: confirmatory factor analysis. Journal of Personality and Social Psychology, 71, 1037-1054.

Sarriera, J.C., Schwarcz, C. \& Câmara, S.G. (1996). Bem-estar psicológico: análise fatorial da escala de Goldberg (GHQ-12) numa amostra de jovens. Psicologia: Reflexão e Crítica, 9, 293-306.

Sheldon, K.M. \& Kasser, T. (1995). Coherence and congruence: Two aspects of personality integration. Journal of Personality and Social Psychology, 68, 531-543.

Tarnopolsky, A., Hand, D.J., Mclean, E.K., Roberts, H. \& Wiggins, R.D. (1979). Validity and uses of a screening questionnaire (GHQ) in the community. British Journal of Psychiatry, 134, 508-515.

Wagner, A., Ribeiro, L.S., Arteche, A.X. \& Bornholdt, E.A. (1999). Configuração familiar e o bem-estar psicológico dos adolescentes. Psicologia: Reflexão e Crítica, 12, 147-156. 